Rowan University

Rowan Digital Works

Faculty Scholarship for the College of Science \& Mathematics

2-2009

\title{
Stress-induced changes in the expression of monocytic 及2-integrins: The impact of arousal of negative affect and adrenergic responses to the Anger Recall Interview
}

Jeffrey M. Greeson

Rowan University, greeson@rowan.edu

James G. Lewis

Karen Achanzar

Eugene Zimmerman

Kenneth H. Young

See next page for additional authors

Follow this and additional works at: https://rdw.rowan.edu/csm_facpub

Part of the Health Psychology Commons

\section{Recommended Citation}

Greeson, J., Lewis, J., Achanzar, K., Zimmerman, E., Young, K., \& Suarez, E. (2009). Stress-induced changes in the expression of monocytic beta(2)-integrins: The impact of arousal of negative affect and adrenergic responses to the anger recall interview. Brain Behavior and Immunity, 23(2), 251-256.

This Article is brought to you for free and open access by the College of Science \& Mathematics at Rowan Digital Works. It has been accepted for inclusion in Faculty Scholarship for the College of Science \& Mathematics by an authorized administrator of Rowan Digital Works. 


\section{Authors}

Jeffrey M. Greeson, James G. Lewis, Karen Achanzar, Eugene Zimmerman, Kenneth H. Young, and Edward C. Suarez 


\title{
Stress-induced changes in the expression of monocytic $\beta_{2^{-}}$ integrins: The impact of arousal of negative affect and adrenergic responses to the Anger Recall Interview
}

\author{
Jeffrey M. Greeson ${ }^{a}$, James G. Lewis ${ }^{b}$, Karen Achanzar ${ }^{b}$, Eugene Zimmerman ${ }^{c}$, Kenneth H. \\ Young $^{\mathrm{b}}$, and Edward C. Suarez ${ }^{\mathrm{a},{ }^{*}}$ \\ aDepartment of Psychiatry and Behavioral Sciences, Duke University Medical Center, Durham, \\ NC, USA \\ bDepartment of Pathology, Duke University Medical Center, Durham, NC, USA \\ 'Department of Pharmacology, Duke University Medical Center, Durham, NC, USA
}

\section{Abstract}

Adhesion of circulating monocytes to the vascular endothelium is one of the earliest steps in the development of atherosclerosis. This leukocyte-to-endothelium interaction is mediated in part by $\beta_{2}$-integrins, a group of cell adhesion molecules that bind to endothelial ligands. Given the significance of this interaction to atherogenesis, we examined the effects of stress, operationalized as the arousal of negative affect (NA) and cardiovascular and catecholamine responses to the Anger Recall Interview (ARI), on the expression of LFA-1 (CD11a), Mac-1 (CD11b) and p150/95 (CD11c) on circulating monocytes (CD14+). Subjects were 173 healthy, nonsmoking men and women (60\% men, $40 \%$ minorities, aged 18-49 year). Arousal of NA, cardiovascular responses (heart rate [HR], systolic blood pressure [SBP], diastolic blood pressure [DBP]), circulating catecholamines (epinephrine [Epi], norepinephrine [Ne]) and $\beta_{2}$-integrin (CD11/CD18) expression were determined prior to and following the ARI. The principal findings were that the ARI, on average, induced a decrease in monocyte expression of $\beta_{2}$-integrins. However, after adjusting for age, sex, body mass index, exercise status, and baseline level of $\beta_{2}$-integrin expression, those individuals who showed the largest increases in NA, Ne and DBP during the ARI showed an increase in monocyte $\beta_{2}$-integrin expression. Thus, heightened psychological and physiological stress responses induced phenotypic changes in monocytic expression of $\beta_{2}$-integrins that are consistent with the role of monocytes/macrophages in vascular inflammation and increased risk of atherosclerotic cardiovascular disease.

\section{Keywords}

$\beta_{2}$-integrin; Stress; Negative affect; Adrenergic reactivity; Inflammation; Cardiovascular disease risk

\section{Introduction}

Adhesion of circulating monocytes to the vascular endothelium is one of the earliest detectable events in the development of atherosclerosis (Ross, 1999). The firm adherence of circulating monocytes to endothelial cells is mediated by the $\beta_{2}$-integrins, a group of cell

(c) 2008 Elsevier Inc. All rights reserved.

*Corresponding author. Fax: + 919668 6419. suare001@ @c.duke.edu (E.C. Suarez). 
surface receptors characterized by an a-chain (CD11a, -b, -c and -d) noncovalently linked to a $\beta$-chain (CD18) that is identical for all $\beta_{2}$-integrins (Gamberg et al., 1998). Activation of $\beta_{2}$-integrins is induced by cytokines, chemotactic factors and coagulation factors, as well as by binding to cell surface receptors (Carlos and Harlan, 1994). Heightened expression of $\beta_{2^{-}}$ integrins along with their counter-structures on endothelial cells (e.g., ICAM-1 for CD11a and $\mathrm{CD} 1 \mathrm{~b}$ ) promotes transendothelial migration of activated monocytes. This process is then followed by monocytic phagocytosis of intravascular lipids and secretion of proinflammatory cytokines, including interleukin (IL)-6 and tumor necrosis factor (TNF)-a (Blake and Ridker, 2001). Proinflammatory cytokine production and increased expression of vascular cell adhesion molecules (CAMs) results in exacerbated inflammation at the site of the early lesion and eventual progression to atherosclerotic plaque (Best and Gersh, 2001; van der Meer et al., 2002).

Negative affective states and traits such as anger and hostility have been associated with an increased risk of atherosclerotic cardiovascular disease (ACVD) (Rozanski et al., 2005). At this time, however, the underlying mechanisms that account for these associations are not well understood. We and others have posited that the relation of state and trait measures of psychological distress to ACVD may be due, in part, to inflammation (Black, 2006; Kop et al., 2003; Suarez, 2003; Suarez et al., 2004b, 2006) and particularly the effects of stress on monocyte function. Although a number of studies have shown that psychological distress is associated with elevations in inflammatory biomarkers such as IL-6 and C-reactive protein (CRP) (e.g., Kop et al., 2008; Suarez, 2003; Suarez et al., 2002), there is a paucity of evidence for the relation of psychological distress to changes in the expression of CAMs by circulating monocytes ${ }^{1}$. Of those few studies that have examined these associations the results have been equivocal. For example, one study showed that monocytic surface expression of CD11a decreased following a speech stressor (Mills et al., 2003). In contrast, a different study using the same stressor reported no changes in monocytic expression of CD11a (Goebel and Mills, 2000). Both of those studies were limited in a number of important ways, including (a) relatively small sample size, (b) assessment of only one or two $\beta_{2}$-integrins on monocytes, and (c) analysis of mean effects for the stress task without consideration of individual differences in psychological and/or physiological stress reactivity. The current study, therefore, aimed to clarify the effect of acute psychological stress on monocytic $\beta_{2}$-integrin expression by examining individual differences in stressinduced arousal of negative affect (NA), adrenergic and cardiovascular (CV) reactivity as predictors of changes in monocyte cell surface expression of three $\beta_{2}$-integrins (CD11a, $\mathrm{CD} 11 \mathrm{~b}$ and $\mathrm{CD} 11 \mathrm{c})$ in a large sample of apparently healthy adults.

\section{Methods}

\subsection{Participants}

One-hundred and seventy-three healthy adult men and women were recruited from the local community. Demographic characteristics of the sample are provided in Table 1. A detailed description of recruitment and subject entry criteria have been presented elsewhere (Suarez et al., 2004b). Briefly, subjects were recruited via advertisements placed in local newspapers and fliers distributed throughout the community. Inclusion criteria included, age 18-50 years, nonsmoker defined as less than six cigarettes/lifetime, and a negative history or current diagnosis of chronic medical conditions known to influence inflammatory biomarkers. These conditions included asthma, allergies, arthritis, diabetes, cancer,

\footnotetext{
${ }^{1}$ The current study focused solely on monocytes and changes in $\beta_{2}$-integrins. Other studies have examined $\beta_{2}$-integrin expression on circulating lymphocytes. As described in the introduction, our focus on monocytes stems from the integral role in atherosclerotic cardiovascular disease.
} 
hypertension and other cardiovascular conditions as well as psychiatric conditions. Women were required not to have used oral contraceptives 6 months prior to participation.

Two weeks prior to their laboratory sessions, subjects were required to be free of acute infections and recent injuries and to have not undergone medical/dental procedures. Subjects were also required not to use prescription medications and over-the-counter preparations, including low-dose aspirin, for 2-weeks prior to the study. Informed consent was obtained prior to the study. Subjects received $\$ 70$ for their participation. The Institutional Review Board of Duke University Medical Center approved this protocol.

Laboratory sessions for female subjects who were premenopausal were scheduled during the follicular phase (days 5-10) of their menses, a time when ovarian hormones are at their nadir. Confirmation of menstrual cycle phase was determined from blood samples collected on the day of the laboratory study. Samples were assayed for levels of estradiol $\left(\mathrm{E}_{2}\right)$ and progesterone $(\mathrm{P})$.

\subsection{Experimental protocol}

Given our interest in biobehavioral mechanisms of ACVD and the role of NA, the protocol was designed to generate data that would allow us to examine the relationship between arousal of NA and molecular mechanisms implicated in atherosclerosis. Based on the welldocumented relationship between negative affectivity and cardiovascular disease, we employed the Anger Recall Interview (ARI) as the laboratory stressor. A detailed account of the experimental protocol has been published previously (Suarez et al., 2004b).

Briefly, subjects reported to the laboratory between 8:00 and 9:00 am after an overnight fast. Upon arrival, subjects were seated in a reclined position and a 19-gauge butterfly needle was inserted in the antecubital vein of the right arm and a blood pressure cuff was placed on the left arm. After a $30 \mathrm{~min}$ rest period, baseline physiological measures were taken during a 15 min period and this was followed by assessment of negative affect. The 15 min baseline period was followed by a 5 min reading task, a 5 min recovery period, a 5 min ARI, and a 15 min second recovery period. The reading task was used to adjust for any cardiovascular increases associated with vocalization. For the reading task, subjects were asked to read aloud a story about the ocean. To insure minimal emotional arousal, subjects were told that they would not be evaluated for either reading style or comprehension of text. Results of these analyses have been published elsewhere (Suarez et al., 2004b).

The instructions for the ARI directed subjects to verbally describe a personal event that occurred within the past 3 months and that elicited feelings of anger when it occurred and still aroused feelings of anger upon recall. Following a 1 min period of preparation, subjects verbally described the event for approximately $4 \mathrm{~min}$. To assist the subjects in the recall process, the experimenter used prompts such as "How did that make you feel when it happened?"

As previously noted, we and others have shown that the ARI evokes significant arousal of NA (e.g., Boltwood et al., 1993; Gottdiener et al., 2003; Suarez et al., 2004b) as well as robust adrenergic reactivity not only in healthy controls (Suarez et al., 2004b), but also in clinical samples of patients with coronary heart disease (Kop et al., 2008) and those with post-traumatic stress disorder where responses to the ARI indicated reduced parasympathetic system functioning (Hughes et al., 2007). Recent studies have also shown that the ARI can evoke increases in proinflammatory biomarkers, such as CRP, in cardiac patients (Kop et al., 2008) and stimulated production of inflammatory cytokines in healthy controls (Suarez et al., 2006). 
The 15-min recovery period following the ARI allowed for any changes in cell surface markers to manifest since studies have suggested that immune changes may occur 15-30 min after the completion of the task (Segerstrom and Miller, 2004).

\subsection{Measures}

2.3.1. Negative affect-Subjective ratings of NA were obtained using a 10-point Likert scale, with 1 representing "not at alf' and 10 representing "very strong feelings". The following affective states were assessed: agitated, angry, anxious, depressed, frustrated, irritated, nervous, sad, tense, and upset (Suarez et al., 2004b). Ratings were summed to create a total NA score. The range for the total NA score was 10-100 with the lower limit of this range representing no NA arousal and the higher limit representing extreme feelings of NA arousal. Change in arousal of NA was calculated by subtracting baseline NA, collected at the completion of baseline, from NA score collected at the end of the ARI. For both, subjects were asked to rate how they felt during the baseline period and during the ARI.

2.3.2. Blood measures-Peripheral blood samples for the analysis of monocytic $\beta_{2^{-}}$ integrins and catecholamines were collected continuously at a rate of $0.7 \mathrm{ml} / \mathrm{min}$ using a Dakmed Continuous Exfusion Pump (Model ML6, Buffalo, NY) and stored in chilled test tubes containing ethylenediaminetetraacetic acid (EDTA). For catecholamines, samples were obtained for baseline, reading, ARI and recovery periods. Separated plasma aliquots were stored in tubes containing glutathione at $-80{ }^{\circ} \mathrm{C}$ until analysis. Blood samples used to quantify expression of monocytic cell surface markers were collected only during the baseline period and during the last 5 min of the recovery period following the ARI. For the complete study, approximately $40-50 \mathrm{ml}$ of blood were collected for each subject.

Absolute monocyte counts at baseline and post-stressor were determined using an automated hematology analyzer (Sysmex Inc, Muldeleine, Il). Separate blood samples were used to determine cell surface expression on peripheral monocytes using fluorescently labeled antibodies (Becton Dickinson, San Jose, CA). Dual color staining of peripheral blood was achieved using combinations of CD14-phycoerythrin (PE) paired with either CD11a, CD11b or CD11c conjugated to fluoroscein isothiocyanate (FITC). Samples were first incubated for 10 min at room temperature (RT) with the various antibody pairs. Red blood cells were then lysed in BD Lyse (Becton Dickinson, San Jose, CA) for 20 min at RT. The samples were then washed, resuspended in 1xPBS and fixed with $1 \%$ paraformaldehyde. Fluorescent conjugated mouse myeloma immunoglobulins of the same isotype as the CD antibodies were used as controls. Samples were analyzed using dual-color flow cytometry (FACSCAN, Becton Dickinson, Franklin Lanes, NJ) and Cell Quest (ver 3.1). Data used in the analyses were derived by subtracting the mean fluorescent intensity (MFI) values of controls from the MFI of at least 10,000 CD14+ cells.

A single blood sample collected during each period was used to assess catecholamine responses. Peripheral catecholamine concentrations (Epi and Ne) were assessed using radioenzymatic assays. Intra- and inter-assay coefficients of variance for the above assays did not exceed $10 \%$.

2.3.3. Cardiovascular measures-Cardiovascular measures including heart rate (HR), systolic blood pressure (SBP), and diastolic blood pressure (DBP) were taken at 1-min intervals using a Critikon Dinamap vital signs monitor (Model 845 XT, Critikon, Tampa, FL). Means were calculated by averaging 1-min values collected during each study period. Changes scores were calculated by subtracting baseline levels from levels during the ARI. 


\subsection{Data analysis strategy}

Within-subjects effects of the ARI task on arousal of NA, sympathetic activation and $\beta_{2^{-}}$ integrin expression were tested using one-way analysis of variance (ANOVA) conducted on change scores. These analyses were followed with multiple linear regression analyses that were used to test the predictive association between stress-induced changes in the arousal of NA, cardiovascular reactivity (HR, SBP and DBP) and catecholamine (Epi and Ne) responses on the one hand, and changes in circulating monocyte expression of $\beta_{2}$-integrins (CD11a, CD11b and CD11c) on the other. For multiple regression models, age, sex, body mass index (BMI), exercise status (coded as $0=$ no or $1=$ yes) and baseline levels of $\beta_{2^{-}}$ integrins were used as covariates. Exercise status was assessed via the response ("yes" or "no") to the question "Do you exercise on a regular basis?" Alpha was set at .05 (2-tailed). All statistics were performed using SAS version 8.02 (SAS Institute, Cary, NC).

\section{Results}

\subsection{Task effect of the Anger Recall Interview on negative affect, sympathetic activation and $\beta_{2}$-integrin expression}

As shown in Table 2, the ARI evoked significant arousal of negative affect, on average $(\mathrm{F}(1,172)=553.93, p<0001)$. In addition, the ARI produced significant activation of sympathetically-driven cardiovascular responses $(\mathrm{HR}: \mathrm{F}(1,172)=334.70, p<.0001$; SBP: $\mathrm{F}(1,172)=563.70, p<.0001 ; \mathrm{DBP}: \mathrm{F}(1,172)=863.30, p<.0001)$ and peripheral catecholamine levels $($ Epi: $\mathrm{F}(1,172)=53.50, p<.0001 ; \mathrm{Ne}: \mathrm{F}(1,172)=173.50, p<.0001$; see Table 2).

Furthermore, the ARI induced significant mean decreases in $\beta_{2}$-integrin cell surface expression (CD11a: $\mathrm{F}(1,172)=3.87, \mathrm{p}=.05 ; \mathrm{CD} 11 \mathrm{~b}: \mathrm{F}(1,172)=5.32, p<.05 ; \mathrm{CD} 11 \mathrm{c}$ : $\mathrm{F}(1,172)=9.44, p<.01)$, concomitant with a significant mean increase in the number of circulating monocytes $(\mathrm{F}(1,131)=7.60, p<.01)$. Change in circulating monocyte number was not significantly correlated with stress-induced changes in $\beta_{2}$-integrin cell surface density (CD11a: $r=.01, p=.93$; CD11b: $r=.00, p=.99$; CD11c: $r=-.01, p=.91$ ).

\subsection{Regression modeling results}

After adjustment for age, sex, BMI, exercise status and baseline surface marker expression ${ }^{2}$, multiple regression analyses revealed that greater arousal of NA in response to the ARI was positively associated with changes in CD11a $\left(\beta=0.16, p=.02\right.$, total $\left.R^{2}=.24\right), \mathrm{CD} 11 \mathrm{~b}(\beta=$ $0.14, p=.03$, total $\left.R^{2}=.32\right)$ and CD11c $\left(\beta=0.16, p=.005\right.$, total $\left.R^{2}=.51\right)$. To illustrate these associations, we generated $\triangle$ NA quartile groups and plotted mean $\triangle$ MFI adjusted for age, sex, BMI, exercise status and baseline MFI. As shown in Fig. 1, only those subjects who showed the greatest arousal of NA during the ARI relative to baseline NA rating (i.e., those scoring in the highest quartile) responded with increases in $\beta_{2}$-integrin density. The lowest three quartile groups all showed decreases in $\beta_{2}$-integrin expression following the ARI, consistent with the mean ARI task effect of decreased $\beta_{2}$-integrin expression observed using ANOVA.

\footnotetext{
${ }^{2} \mathrm{We}$ also included race as a covariate. The pattern of results was similar. Race was not a significant predictor of stress-induced changes in monocyte surface markers. To identify cases that may have unduly influenced the relationships in regression analyses we conducted leverage analysis (Howell, 1992). Leverage measures the degree that a case is unusual with respect to the predictor variable. In all regression models, we identified cases that surpassed the threshold $\mathrm{h}$ value of 0.12 , as calculated by the equation $3(k=$ $1) / N$ where $k$ is the number of predictors and $N$ is the sample size. Using this cut-off score, we identified only three to five cases out of 173 subjects that may have exerted undue influence. In our study, cases with $h_{\mathrm{i}}>.12$ did not necessarily have an important influence on the regression, but they had the potential for it. Dropping the identified cases did not change the pattern of results or statistical significance for the variables of interest, suggesting that these cases did not exert excessive influence on the regression. Given these results, we present the results of analyses of the full sample.
} 
Stress-induced changes in HR, SBP and Epi did not significantly predict changes in $\beta_{2^{-}}$ integrin expression (data not shown). Stress-induced increases in DBP and Ne, however, were selectively associated with subsets of $\beta_{2}$-integrin expression. Specifically, increases in DBP were significantly and positively associated with density changes in CD11c $(\beta=0.18$,

$p=.01$, total $R^{2}=.48$ ). DBP reactivity, however, was not associated with changes in CD11a and $\mathrm{CD} 11 \mathrm{~b}$.

Stress-induced increases in Ne were significantly and positively associated with changes in CD11c $\left(\beta=0.13, p=.02\right.$, total $\left.R^{2}=.50\right)$ with a similar trend for changes in CD11b expression $\left(\beta=0.11, p=.08\right.$, total $\left.R^{2}=.32\right)$ that did not reach statistical significance. $\mathrm{Ne}$ reactivity was not associated with change in CD11a expression. The observed pattern of associations paralleled that of NA reactivity groups, as the highest $\Delta \mathrm{Ne}$ quartile showed an increase in the expression of $\mathrm{CD} 11 \mathrm{c}$ (adjusted $\triangle \mathrm{CD} 11 \mathrm{c}=1.0(10)$, whereas all other $\mathrm{Ne}$ reactivity groups showed decreases in CD11c expression $(\Delta \mathrm{CD} 11 \mathrm{c}$ : $\mathrm{Q} 1=-35(10) ; \Delta \mathrm{Q} 2=$ -31 (10); and $\Delta \mathrm{Q} 3=-24$ (9), adjusted means for lowest three quartiles).

\section{Discussion}

This study examined the effects of acute stress on changes in monocyte cell surface expression of $\beta_{2}$-integrins in healthy, non-smoking adults. In examining these associations, we specifically examined changes in the arousal of NA, cardiovascular reactivity and adrenergic reactivity to the ARI, an ecologically valid laboratory stressor (Suarez et al., 2004b). The principal findings were that the ARI, on average, induced a decrease in monocyte expression of $\beta_{2}$-integrins. However, individuals who showed the largest increases in NA, Ne and DBP during the ARI showed an increase in monocyte $\beta_{2}$-integrin expression, consistent with greater cellular adhesion to the vascular endothelium (Gamberg et al., 1998). These results were observed in a large sample of apparently healthy men and women with each quartile having approximately $40-44$ subjects. The reported observations were statistically independent of age, sex, BMI, exercise status and baseline $\beta_{2}$-integrin expression. Other potential confounding factors were methodologically controlled. These included smoking history, historical or current clinical disease, medication use, and for women, menstrual cycle phase and oral contraceptive use.

Results further revealed that the ARI task induced an increase in circulating monocyte numbers. This observation is consistent with previously reported data by other investigators who have used either an evaluative speech paradigm (Mills et al., 2003) or an exercise task (Hong and Mills, 2008). The observed stress-induced monocytosis is also consistent with the widely reported leukocytosis following participation in acute psychological stressors (Benschop et al., 1996). We also observed a mean decrease in CD11a expression on circulating monocytes following the ARI. This task-related decrease is consistent with observation by Mills et al. (2003) who showed a similar task-related decrease in CD11a cell surface density on monocytes following the evaluative speech stressor (Mills et al., 2003). The current study extends previous observations by Mills and colleagues by suggesting that, on average, the ARI evoked significant decreases in monocyte expression of two other $\beta_{2^{-}}$ integrins, CD11b and CD11c.

It is important to note that the observation of task-related decreases in surface marker expression does not take into account individual differences in emotional and physiological responses to the task. The assessment of individual differences in stressor appraisal, such as intensity of emotional arousal, is particularly important given the well-established observation that laboratory stressors do not evoke homogeneous physiological responses. In our study, task level analysis suggested that participation in the ARI led to overall mean decreases in integrin expression on circulating monocytes. These results are consistent with 
results of regression analyses, which indicated that most individuals showed a decrease in monocytic $\beta_{2}$-integrin expression. However, employing only a task-related analytic approach failed to identify an important subgroup of individuals (e.g., the top quartile of reactors) that expressed an increase in cell surface markers thought to promote atherosclerotic disease. Surprisingly, most studies that have examined stress-induced changes in cell surface markers have not included individual difference level analysis to accompany task level analysis (e.g., Goebel and Mills, 2000; Mills et al., 2003). The differences in the results of these analytic approaches underscore the need for future studies to incorporate individual difference analyses in order to better understand the role of emotional and physiological reactivity to stress and how these responses may contribute to disease susceptibility (Lovallo and Gerin, 2003).

The findings of the current study extend previous observations in other novel ways. In addition to characterizing immunological/ inflammatory responses to the ARI, a task that has not been frequently used in psychoneuroimmunology studies (e.g., Suarez et al., 2006), the current study is the first to provide empirical evidence of the importance of individual differences in arousal of NA and sympathetically driven physiological responses (i.e., cardiovascular and adrenergic activation) on changes in monocyte expression of $\beta_{2^{-}}$ integrins. Given the role of monocytes in the pathogenesis of atherosclerotic cardiovascular disease, the pattern of observations suggests that stress-induced changes in monocyte cell surface markers represent a pathway whereby negative affect could contribute to increased risk of future cardiovascular disease development.

The current findings may also shed light on the equivocal nature of previous observations describing monocyte surface marker responses to acute laboratory stressors. As noted, studies have reported either decreases (Mills et al., 2003) or no change (Goebel and Mills, 2000) in monocyte cell surface expression of CD11a in response to the evaluative speech stressor. One possible explanation for this inconsistency is that individuals differed in arousal of NA and/or sympathetically-driven physiological responses between the two studies. Another possibility is that small sample size contributed to the mixed results. Based on our findings, it is conceivable that laboratory stressors that evoke significant arousal of NA in only a few subjects produce an overall decrease in surface markers, or perhaps no change at all on average. This possibility is particularly salient to studies that have small samples sizes (e.g., <50) of healthy individuals where it is possible that the number of subjects who exhibit increases in cell surface marker expression is small.

There are a number of plausible explanations for the current findings. One possibility is the influence of cytokines and chemokines in activating peripheral monocytes and upregulating $\beta_{2}$-integrin expression (Johnston and Butcher, 2002). In previous studies, we showed that psychological distress and arousal of NA are associated with both increased expression and production of proinflammatory cytokines and chemokines by peripheral monocytes (Suarez et al., 2003, 2004a; Suarez et al., 2006). Others have shown that proinflammatory cytokines and chemokines can directly stimulate leukocyte activation and firm adhesion to endothelial cells by rapidly mobilizing integrin molecules to the cell surface (Fabbri et al., 1999). Taken together, the data suggest that arousal of NA may initiate a cascade of events that leads to increased cell surface marker expression via production of proinflammatory cytokines and chemokines that, alone or in combination, activate peripheral monocytes.

Another possible explanation is that stress-related changes in leukocyte expression of $\beta_{2^{-}}$ integrins is modulated by catecholamines in conjunction with the level of $\beta$-adrenergic receptor expression and the specific leukocyte subset examined (Carlson, 1997; Mills et al., 1998). $\beta_{2}$-adrenergic receptor stimulation by catecholamines has been shown to upregulate integrin expression via activation of an intracellular signaling pathway that involves a G- 
protein signal-transduction mechanism (Padgett and Glaser, 2003). In our laboratory, we have demonstrated $\beta_{2}$-adrenergic receptor functional dysregulation in response to pharmacologic stimulation among high hostile men (Suarez et al., 1998). Combined, these observations suggest that stress-induced sympathetic outflow in the presence of altered $\beta_{2^{-}}$ adrenergic receptor functioning could lead to increases in cellular adhesion.

Finally, it is also possible that changes in $\beta_{2}$-integrin expression on monocytes are due to a sympathetically mediated increase in circulatory shear stress, which is associated with endothelial cell activation, vascular proinflammatory cytokine and chemokine production, preferential expression of integrins on circulating leukocytes, and extravasation into the vascular wall (Fabbri et al., 1999; Krieglstein and Granger, 2001). Therefore, to the extent that arousal of NA stimulates proinflammatory processes and sympathetic activation, it is conceivable that a number of different, interactive cellular and molecular mechanisms could, in part, explain the relationship between the acute arousal of emotional distress and changes in CAM expression on circulating monocytes.

The participants in the present study were nonsmokers and were free of medications, including those known to influence immune regulation and inflammation. In addition, regression models statistically controlled for the potentially confounding influences of age, sex, BMI, exercise regularity, and baseline level of monocytic $\beta_{2}$-integrin expression. It is possible, however, that unmeasured variables may account, in part, for the observed findings. For instance, it has recently been shown that variations in anticipatory cognitive stress appraisal accounts for monocyte production of proinflammatory cytokines in men following a psychosocial laboratory stressor (Wirtz et al., 2007). Additionally, participants' tendency to ruminate about anger-provoking events may have, in part, accounted for the magnitude of association between the arousal of NA and changes in monocyte $\beta_{2}$-integrin expression following the ARI.

In conclusion, the present investigation provides experimental evidence for a plausible psychoimmunological mechanism underlying the established relationship between higher levels of NA and greater susceptibility to ACVD. Specifically, data revealed that heightened stress-induced arousal of NA during a brief anger recall task was associated with increased expression of $\beta_{2}$-integrins on peripheral blood monocytes, a phenotypic change that is instrumental for the adhesion of monocytes to the endothelium in the early stages of atherosclerosis. These findings further demonstrated that stress-induced sympathetic activation selectively predicted increased monocytic cell surface expression of CAMs associated with vascular inflammation. Future research is warranted to (a) investigate the association between arousal of NA and other CAMs implicated in atherosclerosis, and (b) test the possibility that emotion regulation skills modulate individual differences in stressrelated changes in CAM expression.

\section{References}

Benschop RJ, Rodriguez-Feuerhahn M, Schedlowski M. Catecholamine-induced leukocytosis: early observations, current research, and future directions. Brain, Behavior, and Immunity. 1996; 10:7791.

Best PJ, Gersh BJ. Cell adhesion molecules and inflammation in acute coronary syndromes: markers and emerging risk factors. Eur Heart J. 2001; 22:1155-1159. [PubMed: 11440488]

Black PH. The inflammatory consequences of psychologic stress: relationship to insulin resistance, obesity, atherosclerosis and diabetes mellitus, type II. Med Hypotheses. 2006; 67:879-891. [PubMed: 16781084]

Blake GJ, Ridker PM. Novel clinical markers of vascular wall inflammation. Circ Res. 2001; 89:763771. [PubMed: 11679405] 
Boltwood MD, Taylor CB, Burke MB, Grogin H, Giacomini J. Anger report predicts coronary artery vasomotor response to mental stress in atherosclerotic segments. Am J Cardio. 1993; 72:13611365.

Carlos TM, Harlan JM. Leukocyte-endothelial adhesion molecules. Blood. 1994; 84:2068-2101. [PubMed: 7522621]

Carlson SL. Lymphocyte tracking in the CNS and periphery: Relationships to neural-immune interactions. Brain Behav Immunity. 1997; 11:243-244.

Fabbri M, Bianchi LF, Pardi R. Regulation of lymphocyte traffic by adhesion molecules. Inflamm Res. 1999; 48:239-246. [PubMed: 10391111]

Gamberg CG, Valmu L, Fagerholm S, Kotovuori P, Ihanus E, Tian L, Pessa-Morikawa T. Leukocyte integrins and inflammation. Cell Mol Life Sci. 1998; 54:549-555. [PubMed: 9676574]

Goebel MU, Mills PJ. Acute psychological stress and exercise and changes in peripheral leukocyte adhesion molecule expression and density. Psychosom Med. 2000; 62:664-670. [PubMed: 11020096]

Gottdiener JS, Kop WJ, Hausner E, McCeney MK, Herrington D, Krantz DS. Effects of mental stress on flow-mediated brachial arterial dilation and influence of behavioral factors and hypercholesterolemia in subjects without cardiovascular disease. Am J Cardiol. 2003; 92:687-691. [PubMed: 12972107]

Hong S, Mills PJ. Effects of an exercise challenge on mobilization and surface marker expression of monocyte subsets in individuals with normal vs. elevated blood pressure. Brain Behav Immunity. 2008; 22:590-599.

Howell, DC. Statistical Methods in Psychology. PWS-Kent Publishing Comp; Boston: 1992.

Hughes JW, Dennis MF, Beckham JC. Baroreceptor sensitivity at rest and during stress in women with posttraumatic stress disorder or major depressive disorder. J Trauma Stress. 2007; 20:667-676. [PubMed: 17955541]

Johnston B, Butcher EC. Chemokines in rapid leukocyte adhesion triggering and migration. Semin Immunol. 2002; 14:83-92. [PubMed: 11978080]

Kop HP, Kopp CW, Festa A, Kryzyanowska K, Kriwanek S, Minar E, Roka R, Schernthaner G. Impact of weight loss on inflammatory proteins and their association with the insulin resistance syndrome in morbidly obese patients. Arterioscler, Thromb Vasc Biol. 2003; 23:1042-1047. [PubMed: 12714437]

Kop WJ, Weissman NJ, Zhu J, Bonsall RW, Doyle M, Stretch MR, Glaes SB, Krantz DS, Gottdiener JS, Tracy RP. Effects of acute mental stress and exercise on inflammatory markers in patients with coronary artery disease and healthy controls. Am J Cardiol. 2008; 101:767-773. [PubMed: 18328837]

Krieglstein CF, Granger DN. Adhesion molecules and their role in vascular disease. Am J Hypertens. 2001; 14:44S-55S. [PubMed: 11411765]

Lovallo WR, Gerin W. Psychophysiological reactivity: mechanisms and pathways to cardiovascular disease. Psychosom Med. 2003; 63:36-45. [PubMed: 12554814]

Mills PJ, Ziegler MG, Rehman J, Maisel AS. Catecholamines, catecholamine receptors, cell adhesion molecules, and acute stressor-related changes in cellular immunity. Adv Pharmacol. 1998; 42:587590. [PubMed: 9327970]

Mills PJ, Farag NH, Hong S, Kennedy BP, Berry CC, Ziegler MG. Immune cell CD62L and CD11a expression in response to a psychological stressor in human hypertension. Brain Behav Immunity. $2003 ; 17: 260-267$.

Padgett DA, Glaser R. How stress influences the immune response. Trends in Immunology. 2003; 24:444-448. [PubMed: 12909458]

Ross R. Atherosclerosis--An inflammatory disease. N Engl J Med. 1999; 340:115-126. [PubMed: 9887164]

Rozanski A, Blumenthal JA, Davidson KW, Saab PG, Kubzansky L. The epidemiology, pathophysiology, and management of psychosocial risk factors in cardiac practice: the emerging field of behavioral cardiology. J Am Coll Cardiol. 2005; 45:637-651. [PubMed: 15734605]

Segerstrom SC, Miller GE. Psychological stress and the human immune system: a meta-analytic study of 30 years of inquiry. Psychol Bull. 2004; 130:601-630. [PubMed: 15250815] 
Suarez EC. The joint effect of hostility and depressive symptoms on plasma interleukin-6 in apparently healthy men. Psychosom Med. 2003; 65:523-527. [PubMed: 12883100]

Suarez EC, Sherwood A, Hinderliter AL. Hostility and adrenergic receptor responsiveness: evidence of reduced [beta]-receptor responsiveness in high hostile men. J Psychosom Res. 1998; 44:261267. [PubMed: 9532555]

Suarez EC, Lewis JG, Kuhn CM. The relation of aggression, hostility, and anger to lipopolysaccharide-stimulated tumor necrosis factor (TNF)-a by blood monocytes of normal men. Brain Behav Immunity. 2002; 16:675-684.

Suarez EC, Krishnan RR, Lewis JG. The relation of severity of depressive symptoms to monocyteassociated proinflammatory cytokines and chemokines in apparently healthy men. Psychosom Med. 2003; 65:362-368. [PubMed: 12764208]

Suarez EC, Lewis JG, Krishnan RR, Young KH. Enhanced cytokine and chemokine expressions by blood monocytes to in vitro lipopolysaccharide stimulation are associated with hostility and severity of depressive symptoms in healthy women. Psychoneuroendocrinology. 2004a; 29:11191128. [PubMed: 15219635]

Suarez EC, Saab PG, Llabre MM, Kuhn CM, Zimmerman E. Ethnicity, gender, and age effects on adrenoceptors and physiological responses to emotional stress. Psychophysiology. 2004b; 41:450460. [PubMed: 15102131]

Suarez EC, Boyle SH, Lewis JG, Hall RP, Young KH. Increases in stimulated secretion of proinflammatory cytokines by blood monocytes following arousal of negative affect: The role of insulin resistance as moderator. Brain Behav Immunity. 2006; 20:331-338.

van der Meer IM, de Maat MP, Bots ML, Breteler MM, Meijer J, Kiliaan AJ, Hofman A, Witteman JC. Inflammatory mediators and cell adhesion molecules as indicators of severity of atherosclerosis: the Rotterdam study. Arterioscler, Thromb Vasc Biol. 2002; 22:838-842. [PubMed: 12006399]

Wirtz PH, von Kanel R, Emini L, Suter T, Fontana A, Ehlert U. Variations in anticipatory cognitive stress appraisal and differential proinflammatory cytokine expression in response to acute stress. Brain Behav Immunity. 2007; 21:851-859. 


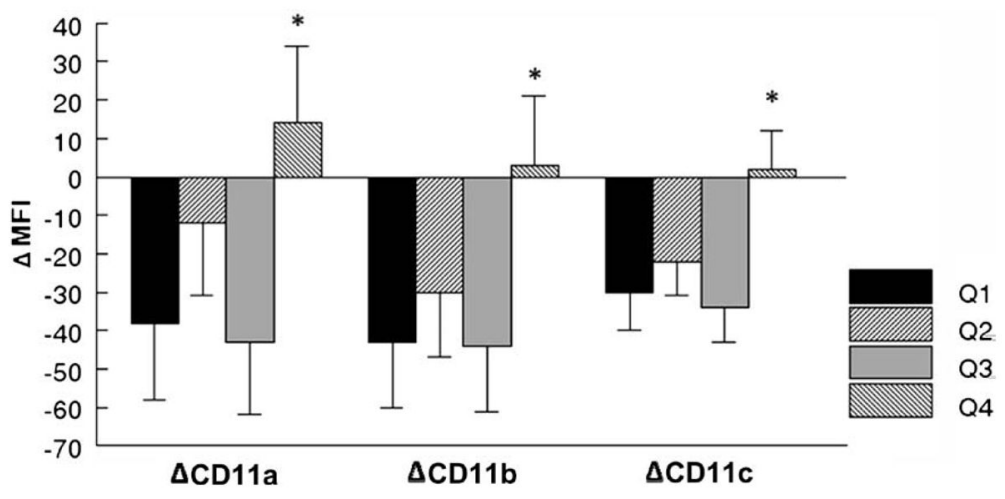

Fig. 1.

Changes in monocyte cell surface expression of $\beta_{2}$-integrins (CD11a, CD11b and CD11c) as a function of stress reactivity, indexed by change in negative affect (Quartiles 1,2,3 and 4) following exposure to the Anger Recall Interview (ARI). Data represent the adjusted change in Mean Fluorescent Intensity (+SD) of monocytic $\beta_{2}$-integrins, controlling for age, sex, body mass index (BMI), exercise status and baseline level. Monocyte cell surface expression of $\beta_{2}$-integrins was significantly greater in the upper quartile range of negative affect (Q4). A similar pattern of findings was observed for norepinephrine (Ne) and diastolic blood pressure (DBP) reactivity (data not shown). ${ }^{*} p<.05$ relative to quartiles 1 and 3 for CD11a and CD11b, and quartiles 1,2 and 3 for CD11c. 
Table 1

Distribution, means (SD) and ranges for demographics of study sample.

\begin{tabular}{|c|c|c|}
\hline & Mean (SD) & Range \\
\hline \multicolumn{3}{|c|}{ Characteristics of study participants } \\
\hline Age (yr) & $26.7(7.7)$ & $18-49$ \\
\hline \multirow[t]{2}{*}{$\operatorname{BMI}\left(\mathrm{kg} / \mathrm{m}^{2}\right)$} & 24.2(1.9) & $16.9-41.8$ \\
\hline & $\mathrm{N}$ & $\%$ \\
\hline \multicolumn{3}{|l|}{ Gender } \\
\hline Male & 104 & 60 \\
\hline Female & 69 & 40 \\
\hline \multicolumn{3}{|l|}{ Race } \\
\hline White & 104 & 60 \\
\hline Black & 40 & 23 \\
\hline Other & 29 & 17 \\
\hline \multicolumn{3}{|l|}{ Exercise regularly } \\
\hline Yes & 142 & 82 \\
\hline No & 31 & 18 \\
\hline \multicolumn{3}{|l|}{ Positive family history } \\
\hline CHD & 22 & 13 \\
\hline Hypertension & 29 & 17 \\
\hline \multicolumn{3}{|l|}{ Educational status } \\
\hline Less than or HS & 5 & 3 \\
\hline Some college or more & 168 & 97 \\
\hline
\end{tabular}


Table 2

Negative affect, sympathetic and monocytic responses to Anger Recall Interview (ARI).

\begin{tabular}{|lllll|}
\hline & Pre-ARI & Post-ARI & F & P \\
\hline Negative affect (units) & $20.5(11.6)$ & $50.7(17.0)$ & 553.93 & $<.0001$ \\
SBP (mmHg) & $136.9(15.9)$ & $154.0(18.1)$ & 563.70 & $<.0001$ \\
DBP (mmHg) & $73.4(9.3)$ & $85.0(9.8)$ & 863.30 & $<.0001$ \\
HR (BPM) & $60.3(8.20)$ & $70.7(9.7)$ & 334.70 & $<.0001$ \\
Ne (pg/ml) & $250.3(95.8)$ & $298.1(108)$ & 173.50 & $<.0001$ \\
Epi (pg/ml) & $29.3(18.4)$ & $39.2(25.0)$ & 53.50 & $<.0001$ \\
CD11a (MFI) & $272.5(241)$ & $253.0(216)$ & 3.87 & $<.05$ \\
CD11b (MFI) & $233.0(235)$ & $211.6(200)$ & 5.32 & $<.05$ \\
CD11c (MFI) & $137.6(137)$ & $119.1(102)$ & 9.44 & $<.01$ \\
CD14 (cells/ $\mu \mathrm{L})$ & $413.2(138)$ & $432.6(144)$ & 7.60 & $<.01$ \\
\hline
\end{tabular}

Note. Data are presented as means (SD). SBP, systolic blood pressure; DBP, diastolic blood pressure; HR, heart rate; Ne, norepinephrine; Epi, epinephrine; CD11a, LFA-1; CD11b, Mac-1; CD11c, gp150/95; MFI, mean fluorescent intensity. 